TRANSACTIONS OF THE

AMERICAN MATHEMATICAL SOCIETY

Volume 350, Number 2, February 1998, Pages 643-664

S $0002-9947(98) 01786-3$

\title{
DERIVATIONS, ISOMORPHISMS, AND SECOND COHOMOLOGY OF GENERALIZED WITT ALGEBRAS
}

\author{
DRAGOMIR Ž. ĐOKOVIĆ AND KAMING ZHAO
}

\begin{abstract}
Generalized Witt algebras, over a field $F$ of characteristic 0 , were defined by Kawamoto about 12 years ago. Using different notations from Kawamoto's, we give an essentially equivalent definition of generalized Witt algebras $W=W(A, T, \varphi)$ over $F$, where the ingredients are an abelian group $A$, a vector space $T$ over $F$, and a map $\varphi: T \times A \rightarrow K$ which is linear in the first variable and additive in the second one.

In this paper, the derivations of any generalized Witt algebra $W=$ $W(A, T, \varphi)$, with the right kernel of $\varphi$ being 0 , are explicitly described; the isomorphisms between any two simple generalized Witt algebras are completely determined; and the second cohomology group $H^{2}(W, F)$ for any simple generalized Witt algebra is computed.

The derivations, the automorphisms and the second cohomology groups of some special generalized Witt algebras have been studied by several other authors as indicated in the references.
\end{abstract}

\section{INTRODUCTION}

Let $p$ be a prime number, $A=\langle x\rangle$ a cyclic group of order $p$, and $F$ a field of characteristic $p$. Then the derivation algebra of the group algebra $F A$ is known as the Witt algebra (see [5, Chapter V, Ex. 21]). It has a basis $\left\{D_{0}, D_{1}, \ldots, D_{p-1}\right\}$ such that $\left[D_{i}, D_{j}\right]=(j-i) D_{i+j}$. Furthermore, it is a simple Lie algebra if $p \neq 2$.

Generalized Witt algebras have been defined by Kaplansky [6] in the context of the classification problem of simple finite dimensional Lie algebras over fields of prime characteristic. His definition is of course applicable also to fields of characteristic 0 . In characteristic 0 case, the term Witt algebra is used as an alternative name for the so-called centerless Virasoro algebra (see e.g. [9]). Amayo and Stewart, in their book [1], refer to this particular algebra as the generalized Witt algebra. The definition of generalized Witt algebras over fields of characteristic 0 has also been given by N. Kawamoto [7]. Although he uses different notations, his definition is actually equivalent to that of Kaplansky.

In this paper we essentially use Kaplansky's definition, slightly generalized. Our notation is different from that of Kawamoto, and can be viewed as a modification of Kaplansky's notation.

Received by the editors January 2, 1996 and, in revised form, April 8, 1996.

1991 Mathematics Subject Classification. Primary 17B40, 17B65; Secondary 17B56, 17B68.

Key words and phrases. Simple Lie algebras, derivations, 2-cocycles, automorphism group.

The first author was supported in part by the NSERC Grant A-5285.

The second author was supported by Academia Sinica of P.R. China.

(c) 1998 American Mathematical Society 
The basic simplicity theorem in the context of generalized Witt algebras of characteristic 0 was proved by Kawamoto [7], although we would not be surprised if it was already known to Kaplansky at the time when he wrote his short note [6].

In spite of more than 40 years since these algebras were defined and studied, the basic isomorphism problem for the simple generalized Witt algebras (of characteristic 0) has remained unresolved. This problem is mentioned briefly in the recent papers $[3,8]$.

We shall now describe the contents and main results of our paper.

In Section 2 we introduce the definition of generalized Witt algebras $W=$ $W(A, T, \varphi)$ where $A$ is an abelian group, $T$ is a vector space over a field $F$, and $\varphi: T \times A \rightarrow F$ a map which is linear in the first variable and additive in the second variable. We also give the classical example of such $W$, from which we have derived our notations in the general case. We describe the center of $W$ and restate Kawamoto's simplicity theorem. Roughly speaking, it says that if $\operatorname{char} F=0$, then $W$ is simple if and only if $A \neq 0$ and $\varphi$ is nondegenerate. We also show that, for a nonzero torsion-free abelian group $A$ of finite rank, $\operatorname{Der}(F A)$ is a simple Lie algebra (and a generalized Witt algebra).

In Section 3 we present two results of R. Farnsteiner [2] on derivations of graded Lie algebras, in a slightly more general form. Then we show how these results can be used to obtain a quick proof of a recent result of Ikeda and Kawamoto [4]. The main result of this section is Theorem 3.4, which gives explicit description of all derivations of $W$ (assuming that the right kernel of $\varphi$ is 0 ). The special case of this theorem, namely when $A$ is a free abelian group of finite rank, was proved recently by Ikeda [3].

In Section 4 we study the structure of isomorphisms $\theta$ between two simple generalized Witt algebras $W=W(A, T, \varphi)$ and $W^{\prime}=W\left(A^{\prime}, T^{\prime}, \varphi^{\prime}\right)$. The main result of this section is Theorem 4.2, which gives an explicit description of all such isomorphisms $\theta$. Hence this theorem provides a solution of the isomorphism problem for simple generalized Witt algebras.

In Section 5 we apply the isomorphism theorem to the study of the automorphism group of $W$. We apply the description of $\operatorname{Aut}(W)$ given by Theorem 5.1 to a special case and obtain a generalization of a recent theorem of Kawamoto [8].

In the last section, Section 6, we assume that $W$ is simple and compute the second cohomology group $H^{2}(W, F)$. This can be viewed as a generalization of the well known fact that the Virasoro algebra is the universal central extension of the Witt algebra. It turns out that $H^{2}(W, F)=0$ if $\operatorname{dim} T \geq 2$, and it is 1-dimensional if $\operatorname{dim} T=1$. In the case $A=\mathbf{Z}^{n}$, assuming only that the right kernel of $\varphi$ is 0 (and so $W$ may be non-simple), $H^{2}(W, F)$ has been computed recently by Ikeda [3].

This research was carried out during the second author's visit to the University of Waterloo. He wishes to thank the Department of Pure Mathematics for its hospitality.

\section{Generalized Witt algebras}

Let $A$ be an abelian group, $F$ a field, and $T$ a vector space over $F$. We denote by $F A$ the group algebra of $A$ over $F$. The elements $t^{x}, x \in A$, form a basis of this algebra, and the multiplication is defined by $t^{x} t^{y}=t^{x+y}$. We shall write 1 instead of $t^{0}$. The tensor product $W=F A \otimes_{F} T$ is a free left $F A$-module. We shall usually denote an arbitrary element of $T$ by $\partial$, to remind us of differential operators (see 
Example 1 below). For the sake of simplicity, we shall write $t^{x} \partial$ instead of $t^{x} \otimes \partial$. We now fix a pairing $\varphi: T \times A \rightarrow F$, which is $F$-linear in the first variable and additive in the second one. For convenience we shall often also use the following notations:

$$
\varphi(\partial, x)=\langle\partial, x\rangle=\partial(x)
$$

for arbitrary $\partial \in T$ and $x \in A$.

There is a unique $F$-bilinear map $W \times W \rightarrow W$ sending $\left(t^{x} \partial_{1}, t^{y} \partial_{2}\right)$ to

$$
\left[t^{x} \partial_{1}, t^{y} \partial_{2}\right]:=t^{x+y}\left(\partial_{1}(y) \partial_{2}-\partial_{2}(x) \partial_{1}\right)
$$

for arbitrary $x, y \in A$ and $\partial_{1}, \partial_{2} \in T$. It is easy to verify that this map makes $W$ into a Lie algebra. We shall refer to $W=W(A, T, \varphi)$ as a generalized Witt algebra.

We now introduce an $A$-gradation of $W$ by setting $W_{x}=t^{x} T$ for $x \in A$. This gradation is compatible with the Lie algebra structure, i.e., $\left[W_{x}, W_{y}\right] \subset W_{x+y}$ for all $x, y \in A$. In particular we have $W_{0}=T$.

It follows from (2.1) that

$$
\left[\partial, t^{x} \partial_{1}\right]=\partial(x) t^{x} \partial_{1},
$$

i.e., $\operatorname{ad}(\partial)$ acts on $W_{x}$ as the scalar $\partial(x)$. Hence $\partial$ is semisimple in the sense that $\operatorname{ad}(\partial)$ is a semisimple operator. Consequently $T$ is a torus, i.e., an abelian subalgebra of $W$ consisting of semisimple elements.

The right kernel of $\varphi$ is the subgroup $A_{0} \subset A$ defined by

$$
A_{0}=\{x \in A:\langle\partial, x\rangle=0, \forall \partial \in T\} .
$$

The left kernel of $\varphi$ is the subspace $T_{0} \subset T$ defined by

$$
T_{0}=\{\partial \in T:\langle\partial, x\rangle=0, \forall x \in A\} .
$$

We say that $\varphi$ is nondegenerate if $A_{0}=0$ and $T_{0}=0$, i.e., if

$$
\langle\partial, x\rangle=0, \forall \partial \in T \Longrightarrow x=0
$$

and

$$
\langle\partial, x\rangle=0, \forall x \in A \Longrightarrow \partial=0 .
$$

Our definition of generalized Witt algebras is slightly more general than that of Kaplansky [6] (ignoring the fact that he assumes that characteristic of $F$ is not 0). The difference is that he assumes that (2.2) holds, and consequently he can identify $x \in A$ with the linear functional on $T$ sending $\partial \rightarrow\langle\partial, x\rangle$.

Kawamoto [7] also assumes that (2.2) is satisfied although he uses different notation. He assumes that $A$ is a subgroup of the direct product

$$
\prod_{i \in I} F_{i}, \quad F_{i}=F
$$

He then defines $W$ to be a vector space with basis consisting of all symbols $w(x, i)$ with $x \in A$ and $i \in I$, and defines Lie multiplication in $W$ by

$$
[w(x, i), w(y, j)]=y_{i} w(x+y, j)-x_{j} w(x+y, i),
$$

where $x_{j}$ is the $j$-th coordinate of $x$, and $y_{i}$ the $i$-th coordinate of $y$. It is now easy to see that his $W$ is also a generalized Witt algebra in our sense: we have only to identify $w(x, i)$ with $t^{x} \partial_{i}$, where $\partial_{i}, i \in I$, is a basis of $T$, and to define $\partial_{i}(x)=x_{i}$, $i \in I$. If $\langle\partial, x\rangle=0$ for all $\partial \in T$, then in particular $x_{i}=\left\langle\partial_{i}, x\right\rangle=0$ for all $i \in I$, 
and so $x=0$. Hence the condition (2.2) is automatically satisfied by Kawamoto's algebras.

It is easy to determine the center $Z$ of an arbitrary generalized Witt algebra $W=W(A, T, \varphi)$.

Lemma 2.1. $Z=F A_{0} \otimes_{F} T_{0}$.

Proof. Let $z \in Z$ and write $z=\sum t^{x} \partial_{x}$, where almost all $\partial_{x} \in T$ are 0 . Clearly each $t^{x} \partial_{x} \in Z$. Assume that $\partial_{x} \neq 0$; then $\left[\partial, t^{x} \partial_{x}\right]=\partial(x) t^{x} \partial_{x}=0$ implies that $\partial(x)=0$ for all $\partial \in T$, i.e., $x \in A_{0}$. It follows that $\left[t^{x} \partial_{x}, t^{y} \partial_{x}\right]=\partial_{x}(y) t^{x+y} \partial_{x}=0$, i.e., $\partial_{x}(y)=0$ for all $y \in A$. Consequently $\partial_{x} \in T_{0}$. Hence we have shown that $Z \subset F A_{0} \otimes_{F} T_{0}$. The opposite inclusion is obvious.

Let $\bar{A}=A / A_{0}$ and let $K$ be the kernel of the natural homomorphism $F A \rightarrow F \bar{A}$. Thus $K$ is spanned by all elements $t^{x}\left(1-t^{y}\right)$ with $x \in A$ and $y \in A_{0}$. It is easy to check that $K T=K \otimes T$ is an ideal of $W$ and $W / K T \simeq W(\bar{A}, T, \bar{\varphi})$, where $\bar{\varphi}: T \times \bar{A} \rightarrow F$ is induced by $\varphi$.

The subspace $F A \otimes T_{0}$ is also an ideal of $W$. It has a complementary subalgebra in $W$, namely $F A \otimes T_{1}$, where $T_{1}$ is a vector space complement of $T_{0}$ in $T$. Furthermore

$$
F A \otimes T_{1}=W\left(A, T_{1}, \varphi_{1}\right),
$$

where $\varphi_{1}: T_{1} \times A \rightarrow F$ is the restriction of $\varphi$.

Hence if $W$ is a simple Lie algebra, then $A \neq 0$ and $\varphi$ must be nondegenerate.

The following theorem is due to Kawamoto [7].

Theorem 2.2. Suppose that characteristic of $F$ is 0 . Then $W=W(A, T, \varphi)$ is a simple Lie algebra if and only if $A \neq 0$ and $\varphi$ is nondegenerate.

According to Kaplansky [6], the analogous result is also valid over fields of finite characteristic, with one exception.

We shall assume from now on that the characteristic of $F$ is 0 . Hence if $\varphi$ satisfies the condition (2.3), then $A$ must be torsion-free.

Example 1. Let $A=\mathbf{Z}^{n}$ and let $\left\{\varepsilon_{1}, \ldots, \varepsilon_{n}\right\}$ be its standard basis. Let us write $t_{i}$ instead of $t^{\varepsilon_{i}}$. Then the group algebra $F A$ becomes identified with the algebra of Laurent polynomials $F\left[t_{1}^{ \pm 1}, \ldots, t_{n}^{ \pm 1}\right]$. Let $T$ be a vector space with basis $\left\{\partial_{1}, \ldots, \partial_{n}\right\}$. Define the pairing $\varphi: T \times A \rightarrow F$ by setting $\left\langle\partial_{i}, \varepsilon_{j}\right\rangle=\delta_{i j}$. The conditions (2.2) and (2.3) are satisfied. The corresponding generalized Witt algebra $W=F A \otimes T$ is simple, and its elements can be written as

$$
P_{1} \partial_{1}+\ldots+P_{n} \partial_{n}
$$

where $P_{i} \in F A$ are Laurent polynomials. If we interpret $\partial_{i}$ as the differential operator $t_{i} \frac{\partial}{\partial t_{i}}$, then we can identify $W$ with the Lie algebra of vector fields with coefficients in the above-mentioned Laurent polynomial ring.

For an arbitrary generalized Witt algebra $W=W(A, T, \varphi)$ we can make the group algebra $F A$ into a left $W$-module. This module structure is characterized by

$$
t^{x} \partial \cdot t^{y}=\partial(y) t^{x+y}
$$

where $x, y \in A$ and $\partial \in T$ are arbitrary.

The $F A$-module structure on $W$ and the $W$-module structure on $F A$ are related by the following identity:

$$
[f u, g v]=f(u \cdot g) v-g(v \cdot f) u+f g[u, v],
$$


where $f, g \in F A$ and $u, v \in W$ are arbitrary.

The $W$-module structure on $F A$ defines a homomorphism of Lie algebras

$$
W \rightarrow \operatorname{Der}(F A)
$$

because each $w \in W$ acts on $F A$ as a derivation. Furthermore it is easy to check that this homomorphism of Lie algebras is also a homomorphism of $F A$-modules.

It is well known that if $F$ is a field of characteristic $0, n$ a positive integer, and $A=\mathbf{Z}^{n}$, then $\operatorname{Der}(F A)$ is a simple Lie algebra. It is natural to ask: what happens if $A$ is an arbitrary abelian group? The next theorem provides a partial answer to that question.

Theorem 2.3. Let $A$ be an abelian group and $F$ a field of characteristic 0 .

(a) If $\operatorname{Der}(F A)$ is a simple Lie algebra over $F$, then $A$ is torsion-free and $A \neq 0$.

(b) If $A$ is torsion-free and $1 \leq \operatorname{rank}(A)<\infty$, then $\operatorname{Der}(F A)$ is simple. Furthermore it is isomorphic to $W(A, T, \varphi)$ for suitably chosen $T$ and $\varphi$.

Proof. (a) Let $A_{t}$ be the torsion subgroup of $A$. If $x \in A_{t}$ then there exists a positive integer $n$ such that $n x=0$. Hence $t^{n x}=1$, and so, if $D \in \operatorname{Der}(F A)$, we have

$$
D\left(t^{n x}\right)=D\left(\left(t^{x}\right)^{n}\right)=n\left(t^{x}\right)^{n-1} D\left(t^{x}\right)=0,
$$

i.e., $D\left(t^{x}\right)=0$. It follows that each $D \in \operatorname{Der}(F A)$ is an $F A_{t}$-linear map, i.e., $\operatorname{Der}(F A)$ is an $F A_{t}$-algebra. If $A_{t} \neq 0$, then $F A_{t}$ is not simple, and consequently $\operatorname{Der}(F A)$ is not simple as a Lie algebra over $F$.

(b) From now on we shall assume that $A_{t}=0$, i.e., $A$ is torsion-free. By Zorn's lemma, we can choose a maximal independent subset $S \subset A$. Let $f: S \rightarrow F A$ be an arbitrary map. We claim that there is a unique derivation $D_{f}$ of $F A$ such that

$$
D_{f}\left(t^{y}\right)=f(y), \quad \forall y \in S .
$$

An arbitrary $x \in A$ can be written uniquely as

$$
x=\sum_{y \in S} c_{x}(y) y,
$$

where $c_{x}(y)$ are rational numbers, almost all 0 . Note that $c_{x}(y) y$ in general is not an element of $A$. Hence the meaning of the above equation is that there exists a positive integer, say $N$, such that all numbers $k_{y}=N c_{x}(y)$ are integers and

$$
N x=\sum_{y \in S} k_{y} \cdot y
$$

holds in $A$. Alternatively one could embed $A$ into the $\mathbf{Q}$-vector space $\mathbf{Q} \otimes \mathbf{Z} A$ so that all the terms $c_{x}(y) y$ acquire the status of vectors in this vector space.

We define

$$
D_{f}\left(t^{x}\right)=\sum_{y \in S} c_{x}(y) f(y) t^{x-y}
$$

and extend it by linearity to obtain a linear map $D_{f}: F A \rightarrow F A$. It is straightforward to verify that $D_{f}$ is a derivation of $F A$. It follows from (2.9) that (2.7) is valid. 
Assume now that $D \in \operatorname{Der}(F A)$ satisfies $D\left(t^{y}\right)=f(y)$ for all $y \in S$. Let $x \in A$ be arbitrary and write it as in (2.8). Choose an integer $n>0$ such that all numbers $k_{y}=n c_{x}(y)$ are integers. By multiplying (2.8) by $n$ and applying $D$, we obtain

$$
D\left(t^{n x}\right)=D\left(\prod_{y \in S} t^{k_{y} y}\right)=\prod_{y \in S} t^{k_{y} y} \cdot \sum_{y \in S} k_{y} t^{-y} D\left(t^{y}\right) .
$$

Since $D\left(t^{n x}\right)=n t^{(n-1) x} D\left(t^{x}\right)$ and $D\left(t^{y}\right)=f(y)$, we conclude that

$$
D\left(t^{x}\right)=\sum_{y \in S} c_{x}(y) f(y) t^{x-y}
$$

Hence $D=D_{f}$, and our claim is proved.

Let $T$ be the vector space over $F$ with basis consisting of all symbols $\partial_{y}, y \in S$. There is a unique pairing $\varphi: T \times A \rightarrow F$ such that

$$
\varphi\left(\partial_{y}, x\right)=c_{x}(y)
$$

for all $x \in A$ and $y \in S$, where the coefficients $c_{x}(y) \in \mathbf{Q}$ are defined by (2.8).

Let $x \in A$ be in the right kernel of $\varphi$. Then $\varphi\left(\partial_{y}, x\right)=c_{x}(y)=0$ for all $y \in S$, and so $x=0$ by $(2.8)$.

Now let $\partial \in T$ be in the left kernel of $\varphi$, and write

$$
\partial=\sum_{y \in S} c_{y} \partial_{y}
$$

where almost all $c_{y} \in F$ are 0 . For $z \in S$ we have

$$
\partial(z)=\sum_{y \in S} c_{y} \partial_{y}(z)=\sum_{y \in S} c_{y} \delta_{y, z}=c_{z},
$$

and so $c_{z}=0$ for all $z \in S$, i.e., $\partial=0$.

Hence we have shown that $\varphi$ is nondegenerate. Consequently the generalized Witt algebra $W=W(A, T, \varphi)$ is a simple Lie algebra provided that $A \neq 0$.

For each $y \in S$ we define $f_{y}: S \rightarrow F A$ by $f_{y}(z)=\delta_{y, z}$. Then for $x \in A$ and $y \in S$ we have

$$
D_{f_{y}}\left(t^{x}\right)=\sum_{z \in S} c_{x}(z) f_{y}(z) t^{x-z}=c_{x}(y) t^{x-y}
$$

and

$$
t^{-y} \partial_{y} \cdot t^{x}=\partial_{y}(x) t^{x-y}=c_{x}(y) t^{x-y} .
$$

Hence the homomorphism (2.6) maps $t^{-y} \partial_{y}$ to $D_{f_{y}}$ for all $y \in S$.

An arbitrary function $f: S \rightarrow F A$ can be written as

$$
f=\sum_{y \in S} F(y) f_{y} .
$$

This makes sense even when $S$ is infinite. Consequently we have

$$
D_{f}=\sum_{y \in S} f(y) D_{f_{y}}
$$

in the sense that for every $u \in F A$ only finitely many $D_{f_{y}}(u), y \in S$, are nonzero and

$$
D_{f}(u)=\sum_{y \in S} f(y) D_{f_{y}}(u)
$$


If $S$ is finite, then the element

$$
w=\sum_{y \in S} f(y) t^{-y} \partial_{y}
$$

is mapped to $D_{f}$ by the homomorphism (2.6), and so this homomorphism is onto.

We conclude that, if $1 \leq|S|<\infty$, then (2.6) is an isomorphism of Lie algebras.

In connection with Theorem 2.3 we raise the following question.

Question 1. If $F$ is a field of characteristic 0 and $A$ a torsion-free group of infinite rank, is it true that $\operatorname{Der}(F A)$ is a simple Lie algebra?

We are not able to answer this question even when $A$ is a free abelian group of countable rank.

\section{Derivations of $W$}

Let $A$ be an abelian group, $L$ an $A$-graded Lie algebra, and $V$ an $A$-graded left $L$-module. We denote by $L_{x}$ resp. $V_{x}$ the homogeneous component of $L$ resp. $V$ of degree $x \in A$.

A linear map $D: L \rightarrow V$ is called a derivation if the equality

$$
D[v, w]=v \cdot D w-w \cdot D v
$$

holds for all $v, w \in L$. If $z \in V$ is fixed, then the linear map $D_{z}: L \rightarrow V$ defined by $D_{z}(w)=w \cdot z$ is a derivation. The derivations $D_{z}$ for $z \in V$ are called inner.

Let $\operatorname{Der}(L, V)$ denote the space of all derivations $L \rightarrow V$ and $\operatorname{Inn}(L, V)$ that of inner derivations. We say that $D \in \operatorname{Der}(L, V)$ has degree $y \in A$ if $D\left(L_{x}\right) \subset V_{x+y}$ for all $x \in A$. We denote by $\operatorname{Der}(L, V)_{x}$ the space of all derivations $L \rightarrow V$ of degree $x$.

We now reformulate, in a slightly more general form, two results of Farnsteiner [2]. In his paper he assumes that $L$ is finitely generated; we drop that assumption. His proofs remain valid for these more general propositions.

Proposition 3.1. Every $D \in \operatorname{Der}(L, V)$ can be written as

$$
D=\sum_{x \in A} D_{x}, \quad D_{x} \in \operatorname{Der}(L, V)_{x}
$$

in the sense that for every $v \in L$ only finitely many $D_{x} v \neq 0$ and

$$
D v=\sum_{x \in A} D_{x} v
$$

A derivation $D: L \rightarrow V$ is locally inner if for every finite set $\left\{v_{1}, \ldots, v_{n}\right\} \subset L$ there exists $x \in V$ such that $D v_{i}=v_{i} \cdot x$ for $i=1, \ldots, n$.

Proposition 3.2. Suppose that the following two conditions hold.

(i) $H^{1}\left(L_{0}, V_{x}\right)=0$ for $x \neq 0$;

(ii) $\operatorname{Hom}_{L_{0}}\left(L_{x}, V_{y}\right)=0$ for $x \neq y$.

Then $\operatorname{Der}(L, V)_{x}, x \neq 0$, consists of inner derivations and consequently

$$
\operatorname{Der}(L, V)=\operatorname{Der}(L, V)_{0}+\operatorname{Der}^{\prime}(L, V),
$$

where $\operatorname{Der}^{\prime}(L, V)$ denotes the space of locally inner derivations $L \rightarrow V$. 
We shall now apply Proposition 3.2 in order to prove the following result of Ikeda and Kawamoto [4].

Proposition 3.3. Let $W=W(A, T, \varphi)$ be a generalized Witt algebra satisfying (2.2). Then

$$
\operatorname{Der}(W)=\operatorname{Der}^{\prime}(W)+\operatorname{Der}(W)_{0},
$$

where $\operatorname{Der}^{\prime}(W)$ is the algebra of locally inner derivations of $W$. If $\operatorname{dim} T<\infty$ then $\operatorname{Der}^{\prime}(W)=\operatorname{ad}(W)$.

Proof. We take $L=V=W$ in Proposition 3.2. For the first assertion, we need only verify that the two conditions of that proposition are satisfied.

The first condition requires that $H^{1}\left(W_{0}, W_{x}\right)=0$ for $x \neq 0$, i.e., that every derivation $D: W_{0} \rightarrow W_{x}$ be inner. For $\partial_{1}, \partial_{2} \in T=W_{0}$ we have $\left[\partial_{1}, \partial_{2}\right]=0$, and consequently $\left[\partial_{1}, D \partial_{2}\right]=\left[\partial_{2}, D \partial_{1}\right]$. As $D \partial_{1}, D \partial_{2} \in W_{x}$, this gives

$$
\partial_{1}(x) D \partial_{2}=\partial_{2}(x) D \partial_{1} \text {. }
$$

As $x \neq 0$ and $W$ satisfies (2.2), we can choose $\partial_{1} \in T$ such that $\partial_{1}(x) \neq 0$. Hence we have

$$
D \partial=\operatorname{ad}(w) \partial, \quad \partial \in T,
$$

where $w=-\partial_{1}(x)^{-1} D \partial_{1} \in W_{x}$. Hence the first condition is satisfied.

Since (2.2) holds, it follows that $W_{x}$ is the root space of $W$ with respect to the torus $T$. Hence if $x \neq y$ we have

$$
\operatorname{Hom}_{T}\left(W_{x}, W_{y}\right)=0,
$$

i.e., the second condition is also satisfied.

In order to prove the second assertion, we assume now that $\operatorname{dim} T=n<\infty$ and we choose a basis $\partial_{1}, \ldots, \partial_{n}$ of $T$. Let $D \in \operatorname{Der}(W)^{\prime}$. By Proposition 3.1, we have

$$
D=\sum_{x \in A} D_{x}
$$

where $D_{x} \in \operatorname{Der}(W)_{x}$. If $x \neq 0$ then, by Proposition $3.2, D_{x}$ is an inner derivation of $W$, and so $D_{x}=\operatorname{ad}\left(t^{x} \partial_{x}\right)$ for some $\partial_{x} \in T$. Let $S \subset A$ consist of all $x \neq 0$ such that $\partial_{x} \neq 0$. Let $S_{i}, i=1, \ldots, n$, consist of all $x \in S$ such that $\partial_{i}(x) \neq 0$. For $x \in S$ we have

$$
D_{x}\left(\partial_{i}\right)=\operatorname{ad}\left(t^{x} \partial_{x}\right)\left(\partial_{i}\right)=-\partial_{i}(x) t^{x} \partial_{x} .
$$

By Proposition 3.1, $D_{x}\left(\partial_{i}\right) \neq 0$ for only finitely many $x \in A$, and so $S_{i}$ is a finite set. Since $S$ is the union of the $S_{i}$ 's, $S$ itself is finite.

It remains to show that $D_{0}$ is also inner. We choose a finite set $R \subset A$ such that

$$
\partial(x)=0, \forall x \in R \quad \Longrightarrow \quad \partial \in T_{0} .
$$

Since $D_{0}$ is locally inner, there exists $\partial \in T$ such that $D_{0}=\operatorname{ad}(\partial)$ on the sum $V$ of $W_{x}$ with $x \in R$. Now let $y \in A$ be arbitrary and set $V^{\prime}=V+W_{y}$. Then there exists $\partial^{\prime} \in T$ such that $D_{0}=\operatorname{ad}\left(\partial^{\prime}\right)$ on $V^{\prime}$. In particular $\operatorname{ad}(\partial)=\operatorname{ad}\left(\partial^{\prime}\right)$ on $V$, and so $\partial^{\prime}-\partial \in T_{0}$. Hence $D=\operatorname{ad}(\partial)$ on the whole algebra $W$.

We shall now describe three kinds of derivations of degree 0 of $W$.

If $\mu: A \rightarrow F$ is any additive map, then the linear map $D_{\mu}: W \rightarrow W$ defined by

$$
D_{\mu}(w)=\mu(x) w, \quad w \in W_{x},
$$

is a derivation of degree 0 . 
Let $u: T \rightarrow T_{0}$ be any linear map, where $T_{0}$ is the left kernel of $\varphi$. Then the linear map $D_{u}: W \rightarrow W$ defined by

$$
D_{u}\left(t^{x} \partial\right)=t^{x} u(\partial), \quad \partial \in T,
$$

is a derivation of degree 0 .

Finally if $\partial_{0} \in T_{0}$ is arbitrary, then the linear map $D_{\partial_{0}}: W \rightarrow W$ defined by

$$
D_{\partial_{0}}\left(t^{x} \partial\right)=\partial(x) t^{x} \partial_{0}, \quad \partial \in T
$$

is also a derivation of degree 0 .

The main result of this section is the next theorem, which gives a description of all derivations of degree 0 of $W$ assuming only that the right kernel of $\varphi$ is 0 . In the special case where $A$ is a free abelian group of finite rank, this theorem was proved by Ikeda [3].

Theorem 3.4. Let $W=W(A, T, \varphi)$ be a generalized Witt algebra such that the right kernel of $\varphi$ is 0 , i.e., such that (2.2) holds. If $D \in \operatorname{Der}(W)_{0}$, then $D$ can be written uniquely as

$$
D=D_{\mu}+D_{u}+D_{\partial_{0}}
$$

for some $\mu \in \operatorname{Hom}(A, F), u \in \operatorname{Hom}_{F}\left(T, T_{0}\right)$, and $\partial_{0} \in T_{0}$.

Proof. For each $x \in A$ define the linear operator $D_{x}: T \rightarrow T$ by

$$
D\left(t^{x} \partial\right)=t^{x} D_{x}(\partial), \quad \partial \in T .
$$

By applying $D$ to $(2.1)$, we obtain

$$
\left[t^{x} D_{x} \partial_{1}, t^{y} \partial_{2}\right]+\left[t^{x} \partial_{1}, t^{y} D_{y} \partial_{2}\right]=t^{x+y}\left(\partial_{1}(y) D_{x+y} \partial_{2}-\partial_{2}(x) D_{x+y} \partial_{1}\right),
$$

i.e.,

$$
\begin{aligned}
& \left\langle D_{x} \partial_{1}, y\right\rangle \partial_{2}-\partial_{2}(x) D_{x} \partial_{1}+\partial_{1}(y) D_{y} \partial_{2}-\left\langle D_{y} \partial_{2}, x\right\rangle \partial_{1} \\
& =\partial_{1}(y) D_{x+y} \partial_{2}-\partial_{2}(x) D_{x+y} \partial_{1} .
\end{aligned}
$$

By setting $y=0$ in (3.1), we conclude that $\left\langle D_{0} \partial_{2}, x\right\rangle=0$ for all $\partial_{2} \in T$ and all $x \in$ $A$. Consequently $D_{0}(T) \subset T_{0}$. Let $u: T \rightarrow T_{0}$ be defined by $u(\partial)=D_{0}(\partial), \partial \in T$. By replacing $D$ with $D-D_{u}$, we may assume that

$$
D_{0}=0 .
$$

By setting $y=-x \neq 0$ in (3.1) and by using (3.2), we obtain the identity

$$
\left\langle D_{x} \partial_{1}, x\right\rangle \partial_{2}+\partial_{2}(x) D_{x} \partial_{1}+\partial_{1}(x) D_{-x} \partial_{2}+\left\langle D_{-x} \partial_{2}, x\right\rangle \partial_{1}=0 .
$$

By evaluating at $x$, we obtain

$$
\left\langle D_{x} \partial_{1}, x\right\rangle \partial_{2}(x)+\left\langle D_{-x} \partial_{2}, x\right\rangle \partial_{1}(x)=0 .
$$

Since $x \neq 0$, we can choose $\partial_{1} \in T$ such that $\partial_{1}(x) \neq 0$. Hence $\partial_{2}(x)=0$ implies that $\left\langle D_{-x} \partial_{2}, x\right\rangle=0$. Consequently there is a constant $\lambda_{x} \in F$ such that

$$
\left\langle D_{-x} \partial, x\right\rangle=-\lambda_{x}\langle\partial, x\rangle, \quad \forall \partial \in T .
$$

By replacing $x$ with $-x$, we obtain

$$
\left\langle D_{x} \partial, x\right\rangle=-\lambda_{-x}\langle\partial, x\rangle, \quad \forall \partial \in T .
$$

By using (3.4) and (3.5), we can rewrite (3.3) as follows:

$$
\partial_{2}(x) D_{x} \partial_{1}+\partial_{1}(x) D_{-x} \partial_{2}=\lambda_{x} \partial_{2}(x) \partial_{1}+\lambda_{-x} \partial_{1}(x) \partial_{2}
$$


Fix $\partial_{2} \in T$ such that $\partial_{2}(x) \neq 0$. Then (3.6) implies that

$$
D_{x} \partial=\lambda_{x} \partial+\partial(x) \partial_{x}, \quad \forall \partial \in T,
$$

where $\partial_{x} \in T$ is defined by

$$
\partial_{x}=\frac{\lambda_{-x} \partial_{2}-D_{-x} \partial_{2}}{\partial_{2}(x)}
$$

The formula (3.7) shows that the expression (3.8) for $\partial_{x}$ is independent of the choice of $\partial_{2} \in T$ provided that $\partial_{2}(x) \neq 0$. Hence (3.8) implies that

$$
D_{-x} \partial=\lambda_{-x} \partial-\partial(x) \partial_{x}, \quad \forall \partial \in T \text {. }
$$

By replacing $x$ by $-x$ and comparing with (3.7), we conclude that

$$
\partial_{-x}=\partial_{x}
$$

By evaluating at $x$, we obtain from (3.7)

$$
\left\langle D_{x} \partial, x\right\rangle=\left(\lambda_{x}+\partial_{x}(x)\right) \partial(x) .
$$

By invoking (3.5), we conclude that

$$
\partial_{x}(x)=-\lambda_{x}-\lambda_{-x}
$$

By replacing $x$ by $-x$ and using (3.9) we obtain

$$
\partial_{x}(x)=\lambda_{x}+\lambda_{-x}
$$

Hence we have

$$
\partial_{x}(x)=0, \quad \lambda_{-x}=-\lambda_{x} .
$$

From (3.7), (3.9), and (3.10) we derive

$$
D_{-x}=-D_{x}
$$

Now assume that $x, y, x+y \neq 0$. By (3.7) for $D_{x}$ and the analogous formula for $D_{y}$ and $D_{x+y}$, the identity (3.1) takes the form

$$
\begin{aligned}
{\left[\Lambda_{x, y} \partial_{2}(x)\right.} & \left.-\partial_{2}(y) \partial_{y}(x)\right] \partial_{1}-\left[\Lambda_{x, y} \partial_{1}(y)-\partial_{1}(x) \partial_{x}(y)\right] \partial_{2} \\
& =\partial_{1}(y) \partial_{2}(y)\left(\partial_{x+y}-\partial_{y}\right)-\partial_{1}(x) \partial_{2}(x)\left(\partial_{x+y}-\partial_{x}\right),
\end{aligned}
$$

where for brevity we have set

$$
\Lambda_{x, y}=\lambda_{x+y}-\lambda_{x}-\lambda_{y} .
$$

By setting $\partial_{1}=\partial_{y}$ and $\partial_{2}=\partial_{x}$, we obtain

$$
\partial_{x}(y) \partial_{y}(x)\left(\partial_{x}-\partial_{y}\right)=0 .
$$

Assume that $\partial_{x} \notin T_{0}$ for some $x \neq 0$. Then there exists $y \in A$ such that $\partial_{x}(y) \neq 0$. If $\partial_{x}=\partial_{y}$, then $\partial_{y}(x)=\partial_{x}(x)=0$ by (3.10). Hence (3.13) implies that $\partial_{y}(x)=0$ in all cases. Since $\partial_{x}(x+y) \neq 0$, we can replace $y$ by $x+y$ in the above argument to conclude that also $\partial_{x+y}(x)=0$. By evaluating both sides of (3.12) at $x$, we now obtain

$$
\partial_{2}(x) \cdot\left[\Lambda_{x, y} \partial_{1}(x-y)+\partial_{1}(x) \partial_{x}(y)\right]=0 .
$$

Since we can choose $\partial_{2}$ such that $\partial_{2}(x) \neq 0$, we conclude that

$$
\Lambda_{x, y} \partial_{1}(x-y)+\partial_{1}(x) \partial_{x}(y)=0 .
$$

By setting $\partial_{1}=\partial_{x}$, we obtain $\Lambda_{x, y}=0$. Hence also $\partial_{1}(x) \partial_{x}(y)=0$. As $\partial_{x}(y) \neq 0$ and $x \neq 0$ while $\partial_{1} \in T$ is arbitrary, we have a contradiction to (2.2). 
Hence we have shown that $\partial_{x} \in T_{0}$ for all $x \neq 0$. Consequently (3.12) now takes the form

$$
\begin{aligned}
\Lambda_{x, y} & \left(\partial_{2}(x) \partial_{1}-\partial_{1}(y) \partial_{2}\right) \\
& =\partial_{1}(y) \partial_{2}(y)\left(\partial_{x+y}-\partial_{y}\right)-\partial_{1}(x) \partial_{2}(x)\left(\partial_{x+y}-\partial_{x}\right) .
\end{aligned}
$$

By evaluating both sides at $x$, we conclude that

$$
\Lambda_{x, y} \partial_{2}(x) \partial_{1}(x-y)=0 .
$$

Since $\partial_{1}, \partial_{2} \in T$ are arbitrary, this equation implies that $\Lambda_{x, y}=0$ if $x, y, x \pm y \neq 0$. By defining $\lambda_{0}=0$ and by invoking (3.10), we infer that

$$
\lambda_{x+y}=\lambda_{x}+\lambda_{y}
$$

for all $x \neq y$. If $x \neq 0$, we have

$$
\lambda_{2 x}=\lambda_{3 x}+\lambda_{-x}=\left(\lambda_{2 x}+\lambda_{x}\right)+\left(\lambda_{x}+\lambda_{-2 x}\right)=2 \lambda_{x} .
$$

Hence (3.15) holds for all $x, y \in A$. Let $\mu: A \rightarrow F$ be the additive map defined by $\mu(x)=\lambda_{x}, x \in A$. By replacing $D$ with $D-D_{\mu}$, we may assume that $\lambda_{x}=0$ for all $x \in A$.

Since (3.15) holds, the equation (3.14) takes the simpler form

$$
\partial_{1}(y) \partial_{2}(y)\left(\partial_{x+y}-\partial_{y}\right)=\partial_{1}(x) \partial_{2}(x)\left(\partial_{x+y}-\partial_{x}\right)
$$

By Lemma 3.5 below, there exists a vector $\partial_{0} \in T_{0}$ such that $\partial_{x}=\partial_{0}$ for all $x \neq 0$. Since $\lambda_{x}=0$ we obtain $D_{x} \partial=\partial(x) \partial_{0}$ from $(3.7)$, and so $D=D_{\partial_{0}}$.

It remains to prove the uniqueness assertion of the theorem. It suffices to show that if

$$
D_{\mu}+D_{u}+D_{\partial_{0}}=0
$$

then $D_{\mu}=D_{u}=D_{\partial_{0}}=0$. By evaluating these derivations at $t^{x} \partial$, we obtain

$$
\mu(x) \partial+u(\partial)+\partial(x) \partial_{0}=0
$$

If $A=0$, then clearly $\mu=0$. Otherwise, since the right kernel of $\varphi$ is 0 , we have $T_{0} \neq T$. Hence we can choose $\partial \in T \backslash T_{0}$, to conclude that $\mu(x)=0$ for all $x$, i.e., $\mu=0$. Consequently $u(\partial)+\partial(x) \partial_{0}=0$. By substituting $2 x$ for $x$, we infer that $u(\partial)=0$ and $D_{\partial_{0}}=0$.

Lemma 3.5. Suppose that a map $A \backslash\{0\} \rightarrow T, x \rightarrow \partial_{x}$, satisfies the equation (3.16) for arbitrary $\partial_{1}, \partial_{2} \in T$. Then this map is necessarily a constant, i.e., there exists $\partial_{0} \in T$ such that $\partial_{x}=\partial_{0}$ for all $x$.

Proof. For $\partial \in T$ let $\hat{\partial} \in \operatorname{Hom}(A, F)$ be defined by $\hat{\partial}(x)=\partial(x)=\langle\partial, x\rangle$.

In order to prove the lemma, it suffices to show that if $x, y \neq 0$ then $\partial_{x}=\partial_{y}$. We first choose $\partial_{2}$ such that $\partial_{2}(x) \partial_{2}(y) \neq 0$. Hence if the ratio $\partial_{1}(y) / \partial_{1}(x)$ depends on the choice of $\partial_{1}$, then (3.16) implies that $\partial_{x+y}=\partial_{x}=\partial_{y}$.

Assume now that the ratio $\partial(y) / \partial(x)$ is independent of the choice of $\partial$ (subject to $\partial(x) \neq 0)$. Then there exists $c \in F^{*}$ such that $\partial(y)=c \partial(x)$ for all $\partial \in T$. We shall distinguish two cases.

Case 1: $\operatorname{dim} T>1$. Let $\hat{\partial}_{1}^{\prime}$ and $\hat{\partial}_{2}^{\prime}$ be linearly independent. Then there exists $z \in A$ such that

$$
\left|\begin{array}{ll}
\partial_{1}^{\prime}(x) & \partial_{1}^{\prime}(z) \\
\partial_{2}^{\prime}(x) & \partial_{2}^{\prime}(z)
\end{array}\right| \neq 0 .
$$


Hence the ratio $\partial(z) / \partial(x)$ depends on the choice of $\partial$. We have seen that this implies that $\partial_{x}=\partial_{z}$. We can replace $x$ with $y$ and obtain similarly that $\partial_{y}=\partial_{z}$. Hence also $\partial_{x}=\partial_{y}$.

Case 2: $\operatorname{dim} \hat{T}=1$. In this case we fix $\partial \in T$ such that $\hat{\partial} \neq 0$. By setting $\partial_{1}=\partial_{2}=\partial$ in (3.16), we obtain the equation

$$
\left[\partial(x)^{2}-\partial(y)^{2}\right] \partial_{x+y}=\partial(x)^{2} \partial_{x}-\partial(y)^{2} \partial_{y} .
$$

By replacing $x$ and $y$ with $k x$ and $l x$, respectively, where $k, l$ are nonzero integers, we obtain the equation

$$
\left(k^{2}-l^{2}\right) d(k+l)=k^{2} d(k)-l^{2} d(l),
$$

where $d(k)=\partial_{k x}$. It follows from (3.18) that $d(k)$ for $k>0$ are uniquely determined by $d(1)$ and $d(2)$. Hence we must have

$$
d(k)=\partial_{0}+\frac{1}{k} \partial_{1}, \quad k>0,
$$

where $\partial_{0}, \partial_{1} \in T_{0}$ are some fixed vectors. By (3.9) we have also

$$
d(-k)=d(k), \quad k>0 .
$$

By setting $k=2$ and $l=-1$ in (3.18), and by using (3.20), we find that $3 d(1)=$ $4 d(2)-d(1)$, i.e., $d(1)=d(2)$. Consequently (3.19) implies that $\partial_{1}=0$.

Hence we have shown that if $x \neq 0$, then

$$
\partial_{k x}=\partial_{x}, \quad k \neq 0 .
$$

If $\partial(x)^{2}=\partial(y)^{2}$, then (3.17) implies that $\partial_{x}=\partial_{y}$. Hence we may assume that $\partial(x)^{2} \neq \partial(y)^{2}$.

By replacing $x$ with $x+y$ in (3.17), we obtain

$$
\partial(x) \partial(x+2 y) \partial_{x+2 y}=\partial(x+y)^{2} \partial_{x+y}-\partial(y)^{2} \partial_{y} .
$$

By substituting $\partial_{x+y}$ from (3.17) into this equation, we obtain

$$
\partial(x-y) \partial(x+2 y) \partial_{x+2 y}=\partial(x) \partial(x+y) \partial_{x}-2 \partial(y)^{2} \partial_{y} .
$$

By replacing $y$ with $2 y$ in (3.17) and using the fact that $\partial_{2 y}=\partial_{y}$, we obtain

$$
\partial(x+2 y) \partial(x-2 y) \partial_{x+2 y}=\partial(x)^{2} \partial_{x}-4 \partial(y)^{2} \partial_{y} .
$$

By eliminating $\partial_{x+2 y}$ from the last two equations, we obtain the equality $\partial_{x}=$ $\partial_{y}$.

\section{The ISOMORPHISM THEOREM}

Let $W=W(A, T, \varphi)$ and $W^{\prime}=W\left(A^{\prime}, T^{\prime}, \varphi^{\prime}\right)$ be two simple generalized Witt algebras. We denote by $X(A)$ the group of characters of $A$, i.e., group homomorphisms $A \rightarrow F^{*}$.

Let $\chi \in X(A), \sigma \in \operatorname{Hom}\left(A, A^{\prime}\right)$, and $\tau \in \operatorname{Hom}_{F}\left(T, T^{\prime}\right)$. Assume that $\sigma$ and $\tau$ satisfy

$$
\langle\tau(\partial), \sigma(x)\rangle=\langle\partial, x\rangle
$$

for all $\partial \in T$ and $x \in A$. Then there is a unique linear map

$$
\theta=\theta(\chi, \sigma, \tau): W \rightarrow W^{\prime}
$$

such that

$$
\theta\left(t^{x} \partial\right)=\chi(x) t^{\sigma(x)} \tau(\partial)
$$


holds for all $\partial \in T$ and $x \in A$. It is straightforward to verify that $\theta$ is a homomorphism of Lie algebras.

If we also assume that $\sigma$ and $\tau$ are isomorphisms, then $\theta$ is an isomorphism. Our main objective is to show that every Lie algebra isomorphism $\theta: W \rightarrow W^{\prime}$ has the form described above.

We say that $w \in W$ is locally finite if the linear operator $\operatorname{ad}(w)$ is locally finite. This means that $W$ is the sum of finite dimensional ad $(w)$-invariant subspaces.

Let $w \in W$ and write $w=\sum w_{x}$, where $w_{x} \in W_{x}=t^{x} T$, and the summation is over all $x \in A$. The support of $w$ is the finite set $S_{w} \subset A$ consisting of all $x$ such that $w_{x} \neq 0$. Assume that a total ordering $\leq$ of $A$ is chosen (compatible with its group structure). If $w \neq 0$ and $y$ is the maximal element of $S_{w}$, then we say that $w_{y}$ is the leading term of $w$.

Lemma 4.1. If $W$ is simple, then $T$ is the set of locally finite elements of $W$.

Proof. It is obvious that the elements of $T$ are locally finite. Let $w \in W \backslash T$. We choose a total ordering $\leq$ of $A$ and let $w_{x}=t^{x} \partial$ be the leading term of $w$. As $w \notin T$, by reversing the ordering of $A$ (if necessary) we may assume that $x>0$.

If $\partial(x) \neq 0$, then the leading term of $\operatorname{ad}(w)^{k}\left(t^{2 x} \partial\right)$ is

$$
k ! \partial(x)^{k} t^{(k+2) x} \partial, \quad k \geq 0 .
$$

Now assume that $\partial(x)=0$. As $\partial \neq 0$, the condition (2.1) implies that there exists $y \in A$ such that $\partial(y) \neq 0$. Then the leading term of $\operatorname{ad}(w)^{k} t^{y} \partial$ is

$$
\partial(y)^{k} t^{y+k x} \partial, \quad k \geq 0
$$

Hence, in both cases, $w$ is not locally finite.

Theorem 4.2. Let $W=W(A, T, \varphi)$ and $W^{\prime}=W\left(A^{\prime}, T^{\prime}, \varphi^{\prime}\right)$ be simple generalized Witt algebras. If $\theta: W \rightarrow W^{\prime}$ is an isomorphism of Lie algebras, then there exist $\chi \in X(A)$, isomorphisms $\sigma: A \rightarrow A^{\prime}$ and $\tau: T \rightarrow T^{\prime}$ satisfying (4.1), such that $\theta=\theta(\chi, \sigma, \tau)$.

Proof. Lemma 4.1 implies that $\theta(T)=T^{\prime}$. Let $\tau: T \rightarrow T^{\prime}$ be the restriction of $\theta$. Clearly $\tau$ is an isomorphism of vector spaces.

Since $\theta(T)=T^{\prime}$, it follows that $\theta$ must map the root spaces of $W$ to the root spaces of $W^{\prime}$. Hence there exists a bijection $\sigma: A \rightarrow A^{\prime}$ such that

$$
\theta\left(W_{x}\right)=W_{\sigma(x)}^{\prime}, \quad x \in A,
$$

and $\sigma(0)=0$.

We claim that $\sigma(x+y)=\sigma(x)+\sigma(y)$ for all $x, y \in A$. It suffices to prove this when $x \neq y$. Then we can choose $\partial \in T$ such that $\partial(x-y) \neq 0$. By applying $\theta$ to

$$
\left[t^{x} \partial, t^{y} \partial\right]=\partial(y-x) t^{x+y} \partial
$$

we conclude that our claim holds. Hence $\sigma: A \rightarrow A^{\prime}$ is an isomorphism.

For each $x \in A$ there is a unique isomorphism $\theta_{x}: T \rightarrow T^{\prime}$ such that

$$
\theta\left(t^{x} \partial\right)=t^{\sigma(x)} \theta_{x}(\partial), \quad \forall \partial \in T .
$$

In particular, we have $\theta_{0}=\tau$. Hence $\tilde{\theta}_{x}:=\tau^{-1} \theta_{x}$ is an automorphism of the vector space $T$. Note that $\tilde{\theta}_{0}$ is the identity operator. By applying $\theta$ to $(2.1)$, we obtain

$$
\left[t^{\sigma(x)} \theta_{x} \partial_{1}, t^{\sigma(y)} \theta_{y} \partial_{2}\right]=t^{\sigma(x+y)} \theta_{x+y}\left(\partial_{1}(y) \partial_{2}-\partial_{2}(x) \partial_{1}\right) .
$$


It follows that

$$
\left\langle\theta_{x} \partial_{1}, \sigma(y)\right\rangle \theta_{y} \partial_{2}-\left\langle\theta_{y} \partial_{2}, \sigma(x)\right\rangle \theta_{x} \partial_{1}=\theta_{x+y}\left(\partial_{1}(y) \partial_{2}-\partial_{2}(x) \partial_{1}\right) .
$$

As $\theta_{x}=\tau \tilde{\theta}_{x}$, we infer that

$$
\left\langle\tau \tilde{\theta}_{x} \partial_{1}, \sigma(y)\right\rangle \tilde{\theta}_{y} \partial_{2}-\left\langle\tau \tilde{\theta}_{y} \partial_{2}, \sigma(x)\right\rangle \tilde{\theta}_{x} \partial_{1}=\partial_{1}(y) \tilde{\theta}_{x+y} \partial_{2}-\partial_{2}(x) \tilde{\theta}_{x+y} \partial_{1} .
$$

By setting $y=0$ and $\partial_{2}=\partial$, we conclude that (4.1) holds. Hence we can rewrite the above equality as

$$
\left\langle\tilde{\theta}_{x} \partial_{1}, y\right\rangle \tilde{\theta}_{y} \partial_{2}-\left\langle\tilde{\theta}_{y} \partial_{2}, x\right\rangle \tilde{\theta}_{x} \partial_{1}=\partial_{1}(y) \tilde{\theta}_{x+y} \partial_{2}-\partial_{2}(x) \tilde{\theta}_{x+y} \partial_{1} .
$$

As $\tilde{\theta}_{0}$ is the identity map, by setting $y=-x \neq 0$, we obtain

$$
\left\langle\tilde{\theta}_{x} \partial_{1}, x\right\rangle \tilde{\theta}_{-x} \partial_{2}+\left\langle\tilde{\theta}_{-x} \partial_{2}, x\right\rangle \tilde{\theta}_{x} \partial_{1}=\partial_{1}(x) \partial_{2}+\partial_{2}(x) \partial_{1} .
$$

By evaluating at $x$, we obtain

$$
\left\langle\tilde{\theta}_{x} \partial_{1}, x\right\rangle\left\langle\tilde{\theta}_{-x} \partial_{2}, x\right\rangle=\partial_{1}(x) \partial_{2}(x) .
$$

By setting $\partial_{1}=\partial_{2}=\partial$, we infer that $\left\langle\tilde{\theta}_{-x} \partial, x\right\rangle=0$ implies that $\partial(x)=0$. It follows that there is a constant $\lambda_{x} \in F^{*}$ such that

$$
\partial(x)=\lambda_{x}\left\langle\tilde{\theta}_{-x} \partial, x\right\rangle, \quad \forall \partial \in T .
$$

By replacing $x$ with $-x$, we conclude that

$$
\partial(x)=\lambda_{-x}\left\langle\tilde{\theta}_{x} \partial, x\right\rangle, \quad \forall \partial \in T .
$$

By using (4.5) and (4.6), we can rewrite (4.4) as

$$
\lambda_{-x}^{-1} \partial_{1}(x) \tilde{\theta}_{-x} \partial_{2}+\lambda_{x}^{-1} \partial_{2}(x) \tilde{\theta}_{x} \partial_{1}=\partial_{1}(x) \partial_{2}+\partial_{2}(x) \partial_{1} .
$$

It follows that

$$
\tilde{\theta}_{x} \partial=\lambda_{x} \partial+\partial(x) \partial_{x}, \quad \forall \partial \in T,
$$

where

$$
\partial_{x}=\lambda_{x} \partial_{2}(x)^{-1} \cdot\left[\partial_{2}-\lambda_{-x}^{-1} \tilde{\theta}_{-x} \partial_{2}\right] \in T
$$

is independent of $\partial_{2}$ provided that $\partial_{2}(x) \neq 0$. Hence (4.8) implies that

$$
\tilde{\theta}_{-x} \partial=\lambda_{-x} \partial-\lambda_{-x} \lambda_{x}^{-1} \partial(x) \partial_{x}, \quad \forall \partial \in T .
$$

By replacing $x$ with $-x$ and comparing the new equation with (4.7), we infer that

$$
\lambda_{x} \partial_{-x}=\lambda_{-x} \partial_{x} .
$$

By evaluating (4.7) at $x$ and using (4.6), we find that

$$
\lambda_{-x} \partial_{x}(x)=1-\lambda_{x} \lambda_{-x} .
$$

By substituting $x$ with $-x$ and using (4.9), we infer that

$$
\lambda_{-x}=\lambda_{x}^{-1}, \quad \partial_{x}(x)=0 .
$$

By using (4.7), we can rewrite (4.3) as

$$
\begin{aligned}
& {\left[\Lambda_{x, y} \partial_{2}(x)-\lambda_{x} \partial_{2}(y) \partial_{y}(x)\right] \partial_{1}-\left[\Lambda_{x, y} \partial_{1}(y)-\lambda_{y} \partial_{1}(x) \partial_{x}(y)\right] \partial_{2}} \\
& =\left[\partial_{1}(y) \partial_{2}(y)-\partial_{1}(x) \partial_{2}(x)\right] \partial_{x+y}+\partial_{1}(x)\left[\lambda_{y} \partial_{2}(x)+\partial_{2}(y) \partial_{y}(x)\right] \partial_{x} \\
& \quad-\partial_{2}(y)\left[\lambda_{x} \partial_{1}(y)+\partial_{1}(x) \partial_{x}(y)\right] \partial_{y},
\end{aligned}
$$


where

$$
\Lambda_{x, y}=\lambda_{x+y}-\lambda_{x} \lambda_{y}
$$

By setting $\partial_{1}=\partial_{x}, \partial_{2}=\partial_{y}$ in (4.11) and by using (4.10), we obtain

$$
\Lambda_{x, y}\left(\partial_{y}(x) \partial_{x}-\partial_{x}(y) \partial_{y}\right)=0 .
$$

Next set $\partial_{1}=\partial_{y}, \partial_{2}=-\partial_{x}$ in (4.11) to obtain

$$
\partial_{x}(y) \partial_{y}(x)\left[\left(\lambda_{y}-\partial_{y}(x)\right) \partial_{x}-\left(\lambda_{x}-\partial_{x}(y)\right) \partial_{y}\right]=0 .
$$

Finally, setting $\partial_{1}=\partial_{2}=\partial_{x}$ in (4.11), we find that

$$
\partial_{x}(y)\left[\partial_{x}(y)\left(\partial_{x+y}-\lambda_{x} \partial_{y}\right)+\left(\Lambda_{x, y}+\lambda_{x} \partial_{y}(x)\right) \partial_{x}\right]=0 .
$$

Suppose that there exists $x \neq 0$ such that $\partial_{x} \neq 0$. Then we can choose $y \in A$ such that $\partial_{x}(y) \neq 0$.

We claim that $\partial_{y}(x)=0$. We prove this claim by contradiction. Thus assume that $\partial_{y}(x) \neq 0$. Then (4.10) implies that $\partial_{x}$ and $\partial_{y}$ are linearly independent. From (4.13) we infer that $\partial_{x}(y)=\lambda_{x}$ and $\partial_{y}(x)=\lambda_{y}$. From (4.12) we obtain that $\Lambda_{x, y}=0$, and (4.14) implies that $\partial_{x+y}=\lambda_{x} \partial_{y}-\lambda_{y} \partial_{x}$. By interchanging $x$ and $y$, we conclude that $\partial_{x+y}=0$ and $\lambda_{x} \partial_{y}=\lambda_{y} \partial_{x}$. This gives $\lambda_{x} \partial_{y}(x)=\lambda_{y} \partial_{x}(x)=0$, a contradiction. Hence we have shown that $\partial_{y}(x)=0$.

As $\partial_{x}(x+y)=\partial_{x}(x)+\partial_{x}(y) \neq 0$, we can replace $y$ by $x+y$ in the above arguments to conclude that also $\partial_{x+y}(x)=0$.

By evaluating (4.11) at $x$, we infer that

$$
\Lambda_{x, y} \partial_{2}(x) \partial_{1}(x-y)+\lambda_{y} \partial_{1}(x) \partial_{2}(x) \partial_{x}(y)=0 .
$$

By setting $\partial_{1}=\partial_{x}$, we conclude that $\Lambda_{x, y}=0$. It follows that $\lambda_{y} \partial_{1}(x) \partial_{2}(x) \partial_{x}(y)=$ 0 . Since $\partial_{1}, \partial_{2} \in T$ are arbitrary, we have a contradiction. Hence we have proved that $\partial_{x}=0$ for all $x \in A, x \neq 0$.

The equation (4.11) now takes the form

$$
\Lambda_{x, y}\left(\partial_{2}(x) \partial_{1}-\partial_{1}(y) \partial_{2}\right)=0 .
$$

By evaluating at $x$, we obtain

$$
\Lambda_{x, y} \partial_{2}(x) \partial_{1}(x-y)=0 .
$$

It follows that $\Lambda_{x, y}=0$, i.e., $\lambda_{x+y}=\lambda_{x} \lambda_{y}$ if $x \neq y$, where $\lambda_{0}=1$ by definition. This suffices in order to conclude that $\lambda_{x+y}=\lambda_{x} \lambda_{y}$ for all $x, y \in A$. Hence the map $\chi: A \rightarrow F^{*}$ defined by $\chi(x)=\lambda_{x}$ is a character.

By (4.7), we obtain

$$
\tilde{\theta}_{x} \partial=\lambda_{x} \partial
$$

and so

$$
\theta\left(t^{x} \partial\right)=\chi(x) t^{\sigma(x)} \tau(\partial) .
$$

Hence we have $\theta=\theta(\chi, \sigma, \tau)$. 


\section{Automorphism group of $W$}

In this section we assume that $W=W(A, T, \varphi)$ is simple. By Theorem 4.2, every $\theta \in \operatorname{Aut}(W)$ has the form $\theta=\theta(\chi, \sigma, \tau)$, where $\chi \in X(A), \sigma \in \operatorname{Aut}(A), \tau \in \mathrm{GL}(T)$, and $\sigma$ and $\tau$ satisfy (4.1). Explicitly, we have

$$
\theta(\chi, \sigma, \tau)\left(t^{x} \partial\right)=\chi(x) t^{\sigma(x)} \tau(\partial)
$$

for all $x \in A$ and $\partial \in T$. By using this formula, we find that

$$
\theta\left(\chi_{1}, \sigma_{1}, \tau_{1}\right) \circ \theta\left(\chi_{2}, \sigma_{2}, \tau_{2}\right)=\theta\left(\left(\chi_{1} \circ \sigma_{2}\right) \chi_{2}, \sigma_{1} \sigma_{2}, \tau_{1} \tau_{2}\right),
$$

and consequently

$$
\theta(\chi, \sigma, \tau)^{-1}=\theta\left(\left(\chi \circ \sigma^{-1}\right)^{-1}, \sigma^{-1}, \tau^{-1}\right) .
$$

The map $X(A) \rightarrow \operatorname{Aut}(W)$ defined by

$$
\chi \rightarrow \theta(\chi, 1,1)
$$

is an injective homomorphism. Let $N$ be the image of this homomorphism. Denote by $\mathcal{A}$ the image of the endomorphism of $\operatorname{Aut}(W)$ defined by $\theta(\chi, \sigma, \tau) \rightarrow \theta(1, \sigma, \tau)$. Its kernel is $N$ and its restriction to $\mathcal{A}$ is the identity map. Hence $\operatorname{Aut}(W)=N \rtimes \mathcal{A}$. Hence we have the following result.

Theorem 5.1. If $W=W(A, T, \varphi)$ is simple, then $\operatorname{Aut}(W) \simeq X(A) \rtimes \mathcal{A}$.

We shall now consider a special case in order to obtain a generalization of a result of Kawamoto [8].

Theorem 5.2. Let $W=W(A, T, \varphi)$ where $A=A_{1} \times \ldots \times A_{n}$, each $A_{i}$ is a nonzero subgroup of $F, T$ is a vector space with basis $\partial_{1}, \ldots, \partial_{n}$, and $\varphi$ is defined by $\varphi\left(\partial_{i}, x\right)=x_{i}, x=\left(x_{1}, \ldots, x_{n}\right) \in A$. Then $W$ is simple and

$$
\operatorname{Aut}(W) \simeq X(A) \rtimes G,
$$

where $G$ is the subgroup of $\mathrm{GL}_{n}(F)$ which consists of the automorphisms $\sigma$ of $F^{n}$ such that $\sigma(A)=A$.

Proof. Theorem 5.1 implies that $\operatorname{Aut}(W) \simeq X(A) \rtimes \mathcal{A}$, and it remains to show that $\mathcal{A} \simeq G$. In order to construct an isomorphism $\mathcal{A} \rightarrow G$, we extend $\varphi$ to the bilinear map

$$
\tilde{\varphi}: T \times F^{n} \rightarrow F
$$

such that $\tilde{\varphi}\left(\partial_{i}, x\right)=x_{i}, x=\left(x_{1}, \ldots, x_{n}\right) \in F^{n}$. Obviously $\tilde{\varphi}$ is nondegenerate. For any linear operator $\tau: T \rightarrow T$ we shall denote by $\tau^{*}$ its adjoint with respect to $\tilde{\varphi}$. Thus we have

$$
\tilde{\varphi}(\tau(\partial), x)=\tilde{\varphi}\left(\partial, \tau^{*}(x)\right)
$$

for all $\partial \in T$ and $x \in F^{n}$.

Now let $\theta=\theta(1, \sigma, \tau) \in A$; thus $\sigma$ and $\tau$ satisfy (4.1). It follows that

$$
\partial(x)=\langle\tau(\partial), \sigma(x)\rangle=\tilde{\varphi}(\tau(\partial), \sigma(x))=\tilde{\varphi}\left(\partial, \tau^{*} \sigma(x)\right),
$$

i.e.,

$$
\tilde{\varphi}\left(\partial, \tau^{*} \sigma(x)-x\right)=0
$$

for all $\partial \in T$ and $x \in A$. It follows that $\tau^{*} \sigma(x)=x$ for all $x \in A$, i.e., $\sigma=\tau^{*-1} \mid{ }_{A}$. It is now easy to verify that the homomorphism $\mathcal{A} \rightarrow G$ defined by $\theta \rightarrow \tau^{*-1}$ is an isomorphism. 
This theorem is a generalization of [8, Theorem 2] because we have dropped the hypothesis that the automorphism $\theta$ is "graded".

\section{Computation of $H^{2}(W, F)$}

In this section we compute the second cohomology group of a simple generalized Witt algebra $W$.

Theorem 6.1. Let $W=W(A, T, \varphi)$ be a simple generalized Witt algebra. If $\operatorname{dim} T \geq 2$, then $H^{2}(W, F)=0$. If $T=F \partial$ is 1-dimensional, then $H^{2}(W, F)$ is 1 -dimensional and is spanned by the cohomology class $[\psi]$, where $\psi: W \times W \rightarrow F$ is the 2-cocycle defined by

$$
\psi\left(t^{x} \partial, t^{y} \partial\right)=\delta_{x+y, 0} \partial(x)^{3}, \quad x, y \in A .
$$

(Note that $\partial$ is a fixed nonzero element of $T$.)

Proof. Let $\psi: W \times W \rightarrow F$ be an arbitrary 2-cocycle. For $x, y \in A$ the map $\psi_{x, y}: T \times T \rightarrow F$ defined by

$$
\psi_{x, y}\left(\partial_{1}, \partial_{2}\right)=\psi\left(t^{x} \partial_{1}, t^{y} \partial_{2}\right)
$$

is bilinear. Since $\psi$ is skew-symmetric, we have

$$
\psi_{x, y}\left(\partial_{2}, \partial_{1}\right)=-\psi_{y, x}\left(\partial_{1}, \partial_{2}\right) .
$$

Since $\psi$ is a 2 -cocycle, we have

$$
\psi\left(\left[t^{x} \partial_{1}, t^{y} \partial_{2}\right], t^{z} \partial_{3}\right)+\psi\left(\left[t^{y} \partial_{2}, t^{z} \partial_{3}\right], t^{x} \partial_{1}\right)+\psi\left(\left[t^{z} \partial_{3}, t^{x} \partial_{1}\right], t^{y} \partial_{2}\right)=0 .
$$

This can be rewritten as

$$
\begin{aligned}
& \partial_{1}(y) \psi_{x+y, z}\left(\partial_{2}, \partial_{3}\right)-\partial_{2}(x) \psi_{x+y, z}\left(\partial_{1}, \partial_{3}\right) \\
& +\partial_{2}(z) \psi_{y+z, x}\left(\partial_{3}, \partial_{1}\right)-\partial_{3}(y) \psi_{y+z, x}\left(\partial_{2}, \partial_{1}\right) \\
& +\partial_{3}(x) \psi_{z+x, y}\left(\partial_{1}, \partial_{2}\right)-\partial_{1}(z) \psi_{z+x, y}\left(\partial_{3}, \partial_{2}\right)=0
\end{aligned}
$$

By setting $y=z=0$, we obtain

$$
\partial_{3}(x) \psi_{x, 0}\left(\partial_{1}, \partial_{2}\right)=\partial_{2}(x) \psi_{x, 0}\left(\partial_{1}, \partial_{3}\right) .
$$

For each nonzero $x \in A$, we choose $\partial_{x} \in T$ such that $\partial_{x}(x)=1$ and define a linear function $f_{x}: T \rightarrow F$ by $f_{x}(\partial)=\psi_{x, 0}\left(\partial, \partial_{x}\right)$. Let $f: W \rightarrow F$ be the linear function such that $f(\partial)=0$ for $\partial \in T$ and $f\left(t^{x} \partial\right)=f_{x}(\partial)$ for $x \neq 0$ and $\partial \in T$. By using (6.4) we deduce that the 2-coboundary

$$
\psi_{f}(u, v)=-f([u, v]), \quad u, v \in W
$$

satisfies

$$
\begin{aligned}
\psi_{f}\left(t^{x} \partial_{1}, \partial_{2}\right) & =-f\left(\left[t^{x} \partial_{1}, \partial_{2}\right]\right)=\partial_{2}(x) f_{x}\left(\partial_{1}\right) \\
& =\partial_{2}(x) \psi_{x, 0}\left(\partial_{1}, \partial_{x}\right)=\psi_{x, 0}\left(\partial_{1}, \partial_{2}\right)
\end{aligned}
$$

for all $\partial_{1}, \partial_{2} \in T$ and $x \neq 0$.

Hence by replacing $\psi$ with the cohomologous 2-cocycle $\psi-\psi_{f}$, we may assume that

$$
\psi_{x, 0}=0, \quad x \neq 0 .
$$

By setting $z=0$ in (6.3) and by using (6.2) and (6.5), we deduce that

$$
\partial_{3}(x+y) \psi_{x, y}\left(\partial_{1}, \partial_{2}\right)=0 .
$$


It follows that

$$
\psi_{x, y}=0, \quad x+y \neq 0 .
$$

It remains to determine the bilinear forms $\psi_{x,-x}$. For simplicity we set

$$
\psi_{x}=\psi_{x,-x} .
$$

From (6.2) we derive

$$
\psi_{x}\left(\partial_{2}, \partial_{1}\right)=-\psi_{-x}\left(\partial_{1}, \partial_{2}\right) .
$$

By setting $z=-x-y$ in (6.3) and using (6.8), we obtain

$$
\begin{aligned}
\psi_{x+y}\left(\partial_{1}(y) \partial_{2}-\partial_{2}(x) \partial_{1}, \partial_{3}\right)= & \psi_{y}\left(\partial_{2}, \partial_{3}(x) \partial_{1}+\partial_{1}(x+y) \partial_{3}\right) \\
& -\psi_{x}\left(\partial_{1}, \partial_{3}(y) \partial_{2}+\partial_{2}(x+y) \partial_{3}\right) .
\end{aligned}
$$

By setting $y=0$ in this equality, we obtain

$$
\psi_{0}\left(\partial_{2}, \partial_{3}(x) \partial_{1}+\partial_{1}(x) \partial_{3}\right)=0
$$

for all $\partial_{1}, \partial_{2}, \partial_{3} \in T$ and $x \in A$. Hence we can conclude that

$$
\psi_{0}=0 .
$$

By setting $\partial_{1}=\partial_{2}=\partial_{3}=\partial$ in (6.9), we obtain

$$
\partial(y-x) \psi_{x+y}(\partial, \partial)=\partial(2 x+y) \psi_{y}(\partial, \partial)-\partial(x+2 y) \psi_{x}(\partial, \partial) .
$$

Hence if $\partial(x) \neq 0$ and $\partial(y)=0$, then

$$
\psi_{x+y}(\partial, \partial)=\psi_{x}(\partial, \partial)-2 \psi_{y}(\partial, \partial) .
$$

Now let $z \in A$ be such that $\partial(z)=0$. By substituting $x+z$ for $x$ in (6.11), we obtain

$$
\partial(y-x) \psi_{x+y+z}(\partial, \partial)=\partial(2 x+y) \psi_{y}(\partial, \partial)-\partial(x+2 y) \psi_{x+z}(\partial, \partial) .
$$

By setting $y=x$, we conclude that

$$
\partial(x) \cdot\left[\psi_{x+z}(\partial, \partial)-\psi_{x}(\partial, \partial)\right]=0 .
$$

If $\partial \neq 0$, then we can choose $x \in A$ such that $\partial(x) \neq 0$, and by invoking (6.12) the previous equality implies that $\psi_{z}(\partial, \partial)=0$.

Hence we have shown that

$$
\partial(z)=0 \quad \Longrightarrow \quad \psi_{z}(\partial, \partial)=0 .
$$

Now let $x \in A$ and $\partial \in T$ be such that $\partial(x) \neq 0$. By substituting $k x$ for $x$ and $l x$ for $y$ in (6.11), we obtain

$$
(k-l) d(k+l)=(k+2 l) d(k)-(2 k+l) d(l),
$$

where

$$
d(k)=\psi_{k x}(\partial, \partial), \quad k \in \mathbf{Z} .
$$

It follows from (6.14) that all the values $d(k)$ can be computed if $d(1)$ and $d(2)$ are known. Hence the general solution of the difference equation (6.14) is given by $d(k)=a k+b k^{3}$, where $a, b \in F$ are arbitrary constants.

By setting $k=1$ and $k=2$, we find that

$$
a=\frac{1}{6}(8 d(1)-d(2)), \quad b=\frac{1}{6}(d(2)-2 d(1)) .
$$


We conclude that

$$
\psi_{k x}(\partial, \partial)=\frac{k}{6}\left[8 \psi_{x}(\partial, \partial)-\psi_{2 x}(\partial, \partial)\right]+\frac{k^{3}}{6}\left[\psi_{2 x}(\partial, \partial)-2 \psi_{x}(\partial, \partial)\right]
$$

for all $k \in \mathbf{Z}$, provided that $\partial(x) \neq 0$.

Note that (6.12) and (6.13) imply that

$$
\psi_{x+y}(\partial, \partial)=\psi_{x}(\partial, \partial)
$$

for all $x, y \in A$ and $\partial \in T$, provided only that $\partial(y)=0$.

We now assume that $\partial \neq 0$ and fix $x_{0} \in A$ such that $\partial\left(x_{0}\right) \neq 0$. Then we claim that

$$
\begin{aligned}
6 \psi_{x}(\partial, \partial)= & {\left[8 \psi_{x_{0}}(\partial, \partial)-\psi_{2 x_{0}}(\partial, \partial)\right] \cdot \frac{\partial(x)}{\partial\left(x_{0}\right)} } \\
& +\left[\psi_{2 x_{0}}(\partial, \partial)-2 \psi_{x_{0}}(\partial, \partial)\right] \cdot\left(\frac{\partial(x)}{\partial\left(x_{0}\right)}\right)^{3}
\end{aligned}
$$

for all $x \in A$.

Assume first that $\partial(x)=k \partial\left(x_{0}\right)$ for some integer $k$. Since $\partial\left(x-k x_{0}\right)=0,(6.17)$ implies that

$$
\psi_{x}(\partial, \partial)=\psi_{k x_{0}}(\partial, \partial)
$$

By setting $x=x_{0}$ in (6.16), we infer that (6.18) is valid.

Now assume that $\partial(x) \notin \mathbf{Z} \cdot \partial\left(x_{0}\right)$. By substituting $2 x_{0}$ for $y$ in (6.11), we obtain

$$
\partial\left(2 x_{0}-x\right) \psi_{x+2 x_{0}}(\partial, \partial)=2 \partial\left(x+x_{0}\right) \partial_{2 x_{0}}(\partial, \partial)-\partial\left(x+4 x_{0}\right) \psi_{x}(\partial, \partial) .
$$

By substituting $x_{0}$ for $x$ and $x+x_{0}$ for $y$ in the same equality, we obtain

$$
\partial(x) \psi_{x+2 x_{0}}(\partial, \partial)=\partial\left(x+3 x_{0}\right) \psi_{x+x_{0}}(\partial, \partial)-\partial\left(2 x+3 x_{0}\right) \psi_{x_{0}}(\partial, \partial) .
$$

By substituting $x_{0}$ for $y$ in (6.11), we obtain

$$
\partial\left(x_{0}-x\right) \psi_{x+x_{0}}(\partial, \partial)=\partial\left(2 x+x_{0}\right) \psi_{x_{0}}(\partial, \partial)-\partial\left(x+2 x_{0}\right) \psi_{x}(\partial, \partial) .
$$

By eliminating $\psi_{x+2 x_{0}}(\partial, \partial)$ and $\psi_{x+x_{0}}(\partial, \partial)$ from the above three equalities, we obtain exactly (6.18). Hence our claim is proved.

Assume now that $\operatorname{dim} T=1$ and fix a nonzero $\partial \in T$. It is easy to check that the bilinear maps $\psi_{1}, \psi_{3}: W \times W \rightarrow F$ defined by

$$
\psi_{i}\left(t^{x} \partial, t^{y} \partial\right)=\delta_{x+y, 0} \partial(x)^{i}, \quad i=1,3,
$$

are 2-cocycles. Furthermore, $\psi_{1}$ is a 2 -coboundary while $\psi_{3}$ is not. The formula (6.18) implies that $\psi$ is a linear combination of $\psi_{1}$ and $\psi_{3}$. Consequently the theorem holds if $\operatorname{dim} T=1$.

From now on we assume that $\operatorname{dim} T \geq 2$. We can write (6.18) in the simplified form

$$
\psi_{x}(\partial, \partial)=a(\partial) \partial(x)+b(\partial) \partial(x)^{3},
$$

where $a(\partial), b(\partial) \in F$ are independent of $x$.

Let $\partial \neq 0$. Assume that there exists $y \in A, y \neq 0$, such that $\partial(y)=0$. Then (6.17) holds for all $x \in A$. Hence by setting $\partial_{1}=\partial_{3}=\partial$ in (6.9), we obtain

$$
\partial_{2}(y) \psi_{x}(\partial, \partial)=2 \partial(x) \psi_{y}\left(\partial_{2}, \partial\right)
$$

whenever $\partial(y)=0$. 
Now we claim that if $\partial \neq 0$ then $b(\partial)=0$ in (6.19). We shall view $\partial$ as a homomorphism $A \rightarrow F$. If $\operatorname{ker}(\partial) \neq 0$, then we can choose $y \neq 0$ such that $\partial(y)=0$, and so (6.20) holds. By fixing $\partial_{2}$ such that $\partial_{2}(y) \neq 0$, our claim follows from (6.20). Assume now that $\operatorname{ker}(\partial)=0$. Since $\operatorname{dim} T \geq 2$, we can choose $\partial^{\prime} \in T$ such that $\partial$ and $\partial^{\prime}$ are linearly independent and both $\partial^{\prime}$ and $\partial+\partial^{\prime}$ have nonzero kernels. Then $b\left(\partial^{\prime}\right)=b\left(\partial+\partial^{\prime}\right)=0$, and so

$$
\begin{gathered}
\psi_{x}\left(\partial^{\prime}, \partial^{\prime}\right)=a\left(\partial^{\prime}\right) \partial^{\prime}(x), \\
\psi_{x}\left(\partial+\partial^{\prime}, \partial+\partial^{\prime}\right)=a\left(\partial+\partial^{\prime}\right) \cdot\left[\partial(x)+\partial^{\prime}(x)\right],
\end{gathered}
$$

for all $x \in A$. We now choose $x \neq 0$ such that $\partial^{\prime}(x)=0$. Then we have

$$
a\left(\partial+\partial^{\prime}\right) \partial(x)=\psi_{x}(\partial, \partial)+\psi_{x}\left(\partial, \partial^{\prime}\right)+\psi_{x}\left(\partial^{\prime}, \partial\right) .
$$

Since $\partial^{\prime}(x)=0,(6.20)$ implies that

$$
\partial(x) \psi_{y}\left(\partial^{\prime}, \partial^{\prime}\right)=2 \partial^{\prime}(y) \psi_{x}\left(\partial, \partial^{\prime}\right)
$$

for all $y \in A$. We choose $y$ such that $\partial^{\prime}(y) \neq 0$. By replacing $x$ with $k x$ in the above equality, we conclude that

$$
\psi_{k x}\left(\partial, \partial^{\prime}\right)=k \psi_{x}\left(\partial, \partial^{\prime}\right), \quad k \in \mathbf{Z} .
$$

By (6.8) we infer that

$$
\psi_{x}\left(\partial^{\prime}, \partial\right)=-\psi_{-x}\left(\partial, \partial^{\prime}\right)=\psi_{x}\left(\partial, \partial^{\prime}\right) .
$$

Hence replacing $x$ with $k x$ in (6.21), we obtain

$$
k a\left(\partial+\partial^{\prime}\right) \partial(x)=\psi_{k x}(\partial, \partial)+2 k \psi_{x}\left(\partial, \partial^{\prime}\right) .
$$

By applying (6.19) to the term $\psi_{k x}(\partial, \partial)$ we obtain

$$
k a\left(\partial+\partial^{\prime}\right) \partial(x)=k a(\partial) \partial(x)+2 k \psi_{x}\left(\partial, \partial^{\prime}\right)+k^{3} b(\partial) \partial(x)^{3} .
$$

Since $k \in \mathbf{Z}$ is arbitrary, we must have $b(\partial)=0$. Hence our claim is proved, i.e., we have

$$
\psi_{x}(\partial, \partial)=a(\partial) \partial(x) .
$$

We define $a(0)=0$, and we claim that $a: T \rightarrow F$ is a linear function. It is immediate from (6.22) that $a(c \partial)=c \cdot a(\partial)$ for $c \in F$. It remains to show that $a\left(\partial+\partial^{\prime}\right)=a(\partial)+a\left(\partial^{\prime}\right)$ when $\partial$ and $\partial^{\prime}$ are linearly independent. From

$$
\psi_{x}\left(\partial+\partial^{\prime}, \partial+\partial^{\prime}\right)=\psi_{x}(\partial, \partial)+\psi_{x}\left(\partial^{\prime}, \partial^{\prime}\right)+\psi_{x}\left(\partial, \partial^{\prime}\right)+\psi_{x}\left(\partial^{\prime}, \partial\right),
$$

by using (6.22), we obtain that

$$
a\left(\partial+\partial^{\prime}\right) \cdot\left\langle\partial+\partial^{\prime}, x\right\rangle=a(\partial) \partial(x)+a\left(\partial^{\prime}\right) \partial^{\prime}(x)+\psi_{x}\left(\partial, \partial^{\prime}\right)+\psi_{x}\left(\partial^{\prime}, \partial\right) .
$$

By replacing $\partial$ with $2 \partial$, we find that

$$
a\left(2 \partial+\partial^{\prime}\right)\left\langle 2 \partial+\partial^{\prime}, x\right\rangle=4 a(\partial) \partial(x)+a\left(\partial^{\prime}\right) \partial^{\prime}(x)+2 \psi_{x}\left(\partial, \partial^{\prime}\right)+2 \psi_{x}\left(\partial^{\prime}, \partial\right) .
$$

By eliminating $\psi_{x}$ from these two equations, we obtain

$$
a\left(2 \partial+\partial^{\prime}\right)\left\langle 2 \partial+\partial^{\prime}, x\right\rangle-2 a\left(\partial+\partial^{\prime}\right)\left\langle\partial+\partial^{\prime}, x\right\rangle=2 a(\partial) \partial(x)-a\left(\partial^{\prime}\right) \partial^{\prime}(x) .
$$

Since $x \in A$ is arbitrary, it follows that

$$
a\left(2 \partial+\partial^{\prime}\right)\left(2 \partial+\partial^{\prime}\right)-2 a\left(\partial+\partial^{\prime}\right)\left(\partial+\partial^{\prime}\right)=2 a(\partial) \partial-a\left(\partial^{\prime}\right) \partial^{\prime} .
$$


Since $\partial$ and $\partial^{\prime}$ are linearly independent, we obtain the equations

$$
\begin{gathered}
a\left(2 \partial+\partial^{\prime}\right)-a\left(\partial+\partial^{\prime}\right)=a(\partial), \\
a\left(2 \partial+\partial^{\prime}\right)-2 a\left(\partial+\partial^{\prime}\right)=-a\left(\partial^{\prime}\right) .
\end{gathered}
$$

By subtracting the second equation from the first, we obtain $a\left(\partial+\partial^{\prime}\right)=a(\partial)+a\left(\partial^{\prime}\right)$. Hence our claim is proved.

Let $g: W \rightarrow F$ be the linear function defined by $g(\partial)=a(\partial), \partial \in T$, and $g\left(t^{x} \partial\right)=0$ for $x \neq 0$ and $\partial \in T$. Let $\psi_{g}$ be the 2-coboundary

$$
\psi_{g}(u, v)=-g([u, v]), \quad u, v, \in W .
$$

We have

$$
\psi_{g}\left(t^{x} \partial_{1}, t^{y} \partial_{2}\right)=\delta_{x+y, 0}\left(a\left(\partial_{1}\right) \partial_{2}(x)+a\left(\partial_{2}\right) \partial_{1}(x)\right),
$$

By using (6.22) and setting $\partial_{1}=\partial_{2}=\partial$, we find that

$$
\psi_{g}\left(t^{x} \partial, t^{y} \partial\right)=2 \delta_{x+y, 0} \psi_{x}(\partial, \partial) .
$$

Hence by replacing $\psi$ with the cohomologous 2 -cocycle $\psi-\frac{1}{2} \psi_{g}$, we may assume that

$$
\psi_{x}(\partial, \partial)=0
$$

for all $x \in A$ and $\partial \in T$. From (6.20) and (6.23) we infer that $\partial(x) \psi_{y}\left(\partial_{2}, \partial\right)=0$ if $\partial(y)=0$. As $x \in A$ is arbitrary, we conclude that $\psi_{y}\left(\partial_{2}, \partial\right)=0$ if $\partial(y)=0$. We can restate this as

$$
\partial^{\prime}(x)=0 \Longrightarrow \psi_{x}\left(\partial, \partial^{\prime}\right)=0 .
$$

It follows from (6.23) that the bilinear forms $\psi_{x}, x \in A$, are skew-symmetric. We claim that they are all zero, i.e.,

$$
\psi_{x}\left(\partial, \partial^{\prime}\right)=0
$$

for all $x \in A$ and all $\partial, \partial^{\prime} \in T$.

If $\partial(x)=0$ or $\partial^{\prime}(x)=0$, then (6.25) follows from (6.24). Otherwise there is a $c \in F^{*}$ such that $\partial(x)=c \partial^{\prime}(x)$. Since $\left\langle\partial-c \partial^{\prime}, x\right\rangle=0,(6.24)$ implies that

$$
\psi_{x}\left(\partial, \partial-c \partial^{\prime}\right)=0 .
$$

By invoking (6.23), we infer that $c \cdot \psi_{x}\left(\partial, \partial^{\prime}\right)=0$. As $c \neq 0$, it follows that (6.25) holds.

Hence our claim is proved, i.e. $\psi_{x}=0$ for all $x \in A$. By invoking (6.6) and (6.7), we conclude that $\psi_{x, y}=0$ for all $x, y \in A$. Hence $\psi=0$ by (6.1).

\section{REFERENCES}

1. R. K. Amayo and I. Stewart, Infinite-dimensional Lie Algebras, Noordhoff, Leyden, 1974. MR 53:570

2. R. Farnsteiner, Derivations and central extensions of finitely generated Lie algebras, J. Algebra 118 (1988), 33-45. MR 89h:17018

3. T. Ikeda, Derivations and central extensions of a generalized Witt algebra, in Nonassociative Algebras and Related Topics, World Scientific, Singapore, 1991, 47-57. MR 93c: 17038

4. T. Ikeda and N. Kawamoto, On the derivations of generalized Witt algebras over a field of characteristic zero, Hiroshima Math. J. 20 (1990), 47-55. MR 91d:17021

5. N. Jacobson, Lie Algebras, Dover, New York, 1979. MR 80k:17001

6. I. Kaplansky, Seminar on simple Lie algebras, Bull. Amer. Math. Soc. 60 (1954), 470-471.

7. N. Kawamoto, Generalizations of Witt algebras over a field of characteristic zero, Hiroshima Math. J. 16 (1986), 417-426. MR 88d:17017 
8. N. Kawamoto, On G-graded automorphisms of generalized Witt algebras, in Contemporary Mathematics, Second International Conference on Algebra (L.A. Bokut, A.I. Kostrikin and S.S. Kutateladze, editors), 184 (1995), 225-230. MR 97b:17017

9. R.V. Moody and A. Pianzola, Lie Algebras with Triangular Decompositions, J. Wiley, New York, 1995. MR 96d:17025

10. J.M. Osborn, New simple infinite dimensional Lie algebras of characteristic 0, J. Algebra 185 (1996), 820-835. CMP 1997\#4

11. J.M. Osborn, Derivations and isomorphisms of Lie algebras of characteristic 0 (preprint).

12. J.M. Osborn, Automorphisms of the Lie algebras $W^{*}$ in characteristic 0 (preprint).

Department of Pure Mathematics, University of Waterloo, Waterloo, Ontario N2L 3G1, CANADA

E-mail address: dragomir@herod.uwaterloo.ca

Institute of Systems Science, Academia Sinica, Beijing, 100080, China

Current address: Department of Mathematics, University of Wisconsin, Madison, Wisconsin 53706-1388

E-mail address: zhao@math.wisc.edu 